\title{
Association and prediction of severe 5-fluorouracil toxicity with dihydropyrimidine dehydrogenase gene polymorphisms: A meta-analysis
}

\author{
HENRY W.C. LEUNG ${ }^{1}$ And AGNES L.F. CHAN ${ }^{2}$
}

Departments of ${ }^{1}$ Radiation Therapy and ${ }^{2}$ Pharmacy, China Medical University-An Nan Hospital, Tainan 709, Taiwan, R.O.C.

Received July 21, 2015; Accepted August 12, 2015

DOI: $10.3892 /$ br.2015.513

\begin{abstract}
The aim of the present study was to evaluate the association and prediction of dihydropyrimidine dehydrogenase gene $(D P Y D)$ polymorphisms and the risk of 5-fluorouracil (5-FU) severe toxicity in cancer patients. A meta-analysis of the published literature was conducted to summarize evidence for DPYD gene polymorphisms associated with an increased risk of severe 5-FU toxicity in patients with cancer from an Asian population. Relevant literature was identified using the PubMed and Cochrane databases on April 11, 2014. Combined risk ratios and $95 \%$ confidence intervals (CIs) were calculated in a fixed-effects model. A total of 5 clinical studies were retrieved in the meta-analysis, including 764 cancer patients with DPYD gene polymorphisms who received 5-FU-based chemotherapy. Overall, DPYD gene polymorphisms were associated with the increased risk of 5-FU severe toxicity [risk ratio=2.54 (2.15-3.00); 95\% CI, 19.46-84.57; $\mathrm{P}=0.0001]$. In conclusion, the present meta-analysis suggested that polymorphisms of several DPYD gene polymorphisms are associated with an increased risk of severe toxic response to 5-FU.
\end{abstract}

\section{Introduction}

Adverse drug reactions to 5-fluorouracil (5-FU)-based chemotherapy have been reported to correlate with dihydropyrimidine dehydrogenase gene $(D P Y D)$ variations in numerous countries. Three genetic variants of thymidylate synthase (TS; encoded by the TYMS gene) and 2 variants of methylenetetrahydrofolate reductase (encoded by the MTHFR gene) are also proposed to be associated with an increased risk of toxicity following 5-FU administration (1). 5-FU has been reported to cause $0.5-1 \%$ mortality $(2,3)$ Therefore, studies have focused on the identification of biomarkers or predictors of 5-FU toxicity $(4,5)$.

Correspondence to: Dr Agnes L.F. Chan, Department of Pharmacy, China Medical University-An Nan Hospital, 66, Section 2, Changhe Road, Tainan, Taiwan, R.O.C.

E-mail: agnes.lf@gmail.com

Key words: dihydropyrimidine dehydrogenase gene polymorphism or variant, 5-fluorouracil-associated toxicity, Asian population
5-FU metabolism involves numerous enzyme reactions and intermediates, however, dihydropyrimidine dehydrogenase (DPD) is the initial and rate-limiting enzyme of the pyrimidine base catabolism, which may increase the half-life of 5-FU-based chemotherapy drugs, thereby increasing the risk of dose-dependent severe toxicity in cancer patients (6). Numerous genetic polymorphisms and rare polymorphisms in FU metabolism have been reported to influence the risk of toxicity, following the first report of severe DPYD deficiency with life- threatening 5-FU toxicity (7-11).

DPD activity varies widely among different human populations; 3-5\% of the general population experiences low or partial DPD deficiency (12-14). Patients with low DPD activity are correlated to a higher risk of developing severe or even lethal toxicity when treated with standard doses of 5-FU (15). This finding was supported by the recently published study from China (16). 5-FU toxicities can be prevented and avoided by dose adjustment when detection of functionally distinct gene polymorphisms can be performed. However, the existing published studies are inconsistent in reporting and testing toxicities. No specificity and validation on several polymorphisms in commercial FU toxicity kits has been reported. Therefore, the detection of candidate gene polymorphisms that are truly associated with FU toxicity becomes uncertain. Numerous studies from Western countries have identified that certain polymorphisms are associated with 5-FU-based chemotherapeutic agents. Recently, there were clinical trials published from an Asian population that investigated the association between DPYD polymorphisms and 5-FU-based chemotherapy toxicity. The present study performed a systematic review and a meta-analysis synthesizing these data to examine its potential use as a biomarker of 5-FU toxicity.

\section{Materials and methods}

Search strategy. A systematic literature search of the PubMed and Cochrane databases was conducted to identify all the clinical studies evaluating types and frequency of DPYD polymorphisms and 5-FU toxicity in cancer patients from Asian populations, including Korea, Japan, China and Thailand, between January 1, 1999 and December 31, 2013. The databases were searched using the medical subject headings or text keywords: Dehydropyrimidine dehydrogenase (DPYD) polymorphism or variant, 5-FU-toxicity, Korean, Japanese, 
Chinese and Thai cancer patients. A manual search was also performed of the references of the selected studies to identify any overlooked literature. Only English language studies were restricted in the search.

Selection criteria. The inclusion criteria were as follows: i) Research focused on the association of DPYD variants with the risk of 5-FU-based treatment toxicity; ii) clinical studies; and iii) available genotype and allele data. Studies were excluded as follows: i) Unpublished studies, conference articles, reviews and duplication of publications; ii) data was unavailable for calculating genotype or allele frequencies (AF); and iii) no data of 5-FU-based toxicity.

Data extraction and synthesis. Two investigators independently selected the studies and extracted data. All the disagreements were resolved by consensus. Abstracts were initially screened to exclude clearly ineligible studies and subsequently the full texts of all the remaining studies were reviewed. A standardized data-recording form was used to summarize data regarding included sequence variations of $D P Y D$, allelic variants, and the frequency and severity of 5-FU-based toxicity in Asian populations.

Statistical analysis. A quantitative meta-analytical technique was used to pool the data for the relative risk (RR) of 5-FU-based toxicity related to DPYD variants. The meta-analysis was performed using the fixed-effects model in Review Manager (Version 5.3; Copenhagen: The Nordic Cochrane Centre, The Cochrane Collaboration, 2014). For the calculation of RR, patients with DPYD gene polymorphisms showed severe toxicity ( $\geq$ grade 2 ) and were compared with those who did not have toxicity in the same trial, and data was extracted directly from the enrolled studies.

Quality assessment. The methodological quality of the included studies was evaluated through ratings on the Newcastle-Ottawa Scale (17). This scale assesses the quality of observational and nonrandomized or cohort studies. The instrument uses a star system to evaluate studies based on 3 criteria: Participant selection, comparability of study groups and assessment of outcome or exposure. Two investigators independently assessed the quality of all the included studies.

Sensitivity analysis. Sensitivity analyses were conducted by changing the fixed or random effects model to estimate the effect on the pooled results. The influence of individual studies on the pooled results was estimated by omitting one study at a time. $\mathrm{P}<0.05$ was considered to indicate a statistically significant difference.

\section{Results}

Literature search. Fig. 1 depicts the flow diagram of the systematic literature search and selection of clinical studies. The systematic literature search identified 51 abstracts. A total of 43 studies were excluded as they did not examine $D P Y D$ variants associated with 5-FU-based toxicity. A total of 5 full-length clinical studies were reviewed and matched the inclusion criteria of the present study $(16,18-21)$.

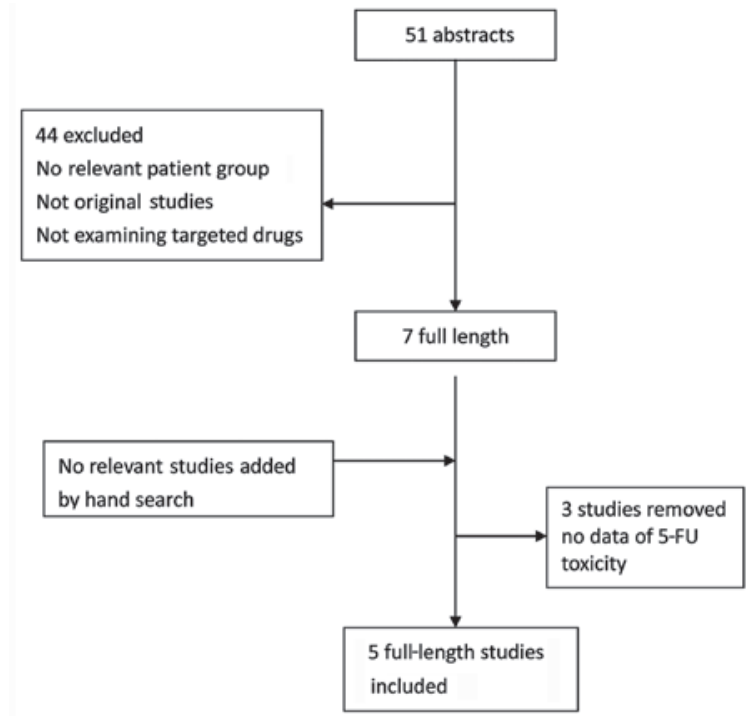

Figure 1. Flow chart of the included studies. 5-FU, 5-fluorouracil

Characteristics of studies. A total of 764 cancer patients with DPYD polymorphisms had received 5-FU-based chemotherapy. Two studies were performed in China, and one each in Japan, Korea and Thailand. A total of 41 single-nucleotide polymorphisms (SNPs) with different AF were detected. Three identical DPYD variants were observed in Chinese and Korean cancer patients: $85 \mathrm{~T}>\mathrm{C}\left(D P Y D^{*} 9 \mathrm{~A}\right), 1627 \mathrm{~A}>\mathrm{G}\left(D P Y D^{*} 5\right)$ and 2194G $>$ A $\left(D P Y D^{*} 6\right)$. The $1896 \mathrm{~T}>\mathrm{C}$ variant was observed in cancer patients of Korea, Japan and Thailand. The $1774 \mathrm{C}>\mathrm{T}$ variant was observed in Korean and Thai patients. The majority of the enrolled studies reported that the AF was $>1 \%$. The AF of the 85T $>$ C variant (Cys29Arg, ${ }^{*} 9 \mathrm{~A}$ ) was similar in Japanese (3.7 and 2.9\%) and Korean patients (2.5\%), but was higher in Chinese patients (7.04\%). The $1627 \mathrm{~A}>\mathrm{G}$ variant was present with comparable AF among Thai (37.07\%), Chinese (20.8\%) and Korean (20.5\%) populations. The AF of $2194 \mathrm{G}>\mathrm{A}$ in the Korean population (1.5\%) was similar to the mean AF of 2 studies of the Chinese population (0.7 and 5\%). Other novel genotypes, such as 496A $>$ G, 74A $>$ G and 1737 T $>C$ were identified in the Korean and Japanese populations with a low AF. The $1896 \mathrm{~T}>\mathrm{C}$ variants were identified in Japanese and Thai populations at frequencies ranging from 9.8 to $15.52 \%$. All the included studies reported an increased risk of severe toxicity ( $\geq$ grade 2 or grades 3 and 4 ) of 5-FU-based chemotherapy that was associated with these common polymorphisms (Table I). The methodological quality of the majority of the studies was moderate (Table II).

Meta-analysis. A meta-analysis of 5 clinical studies showed a significant increased risk of 5-FU-based severe toxicity associated with DPYD gene polymorphisms [risk ratio=2.54 (2.15-3.00); 95\% confidence interval, 19.46-84.57; $\mathrm{P}=0.0001]$ (Fig. 2).

Sensitivity analysis. The result of sensitivity analysis by changing either the fixed effects model to the random effects model or omitting one study at a time indicated no change of the overall risk. Therefore, the present results were deemed statistically reliable. 


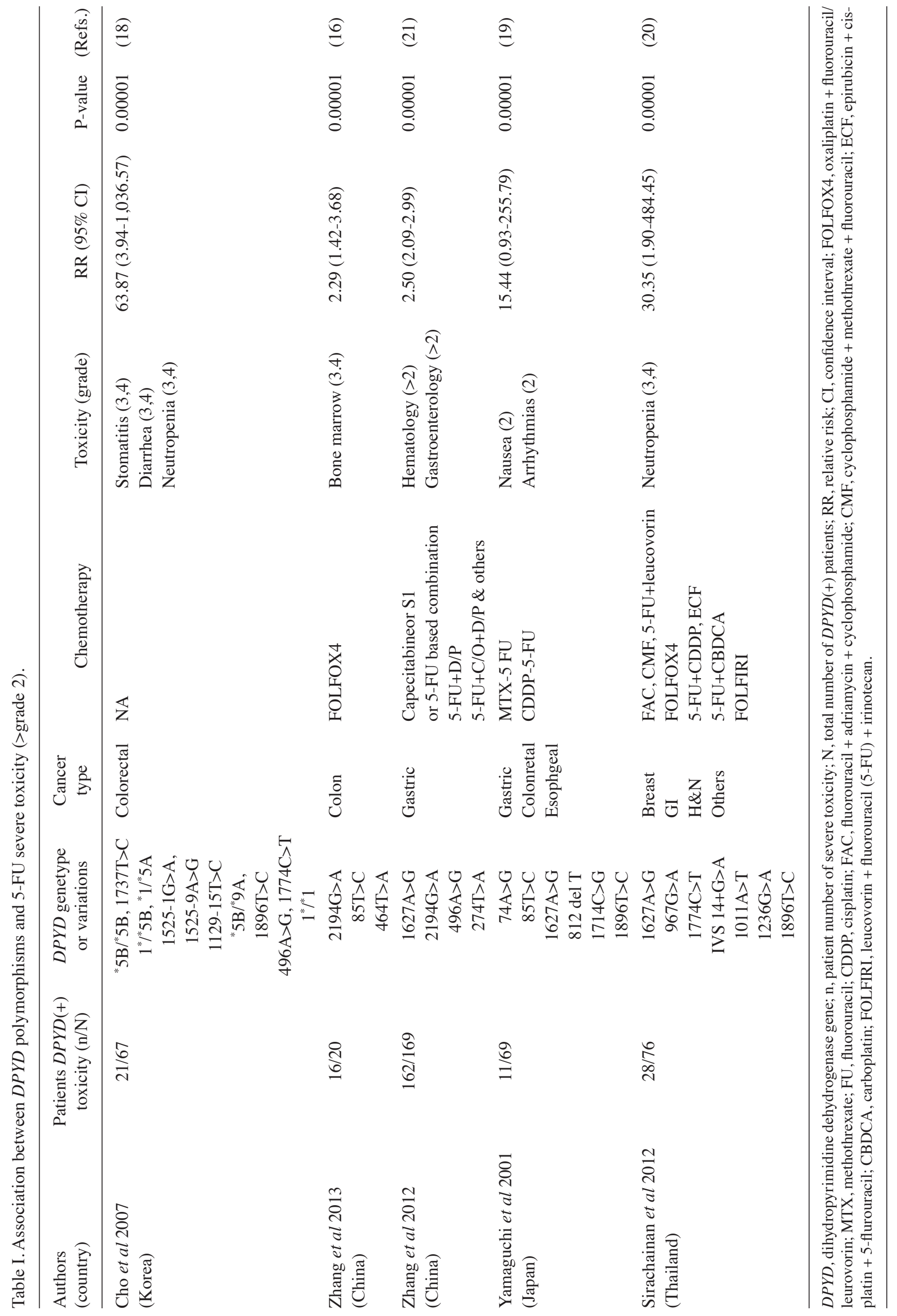


Table II. Assessing the quality of the included studies using the Newcastle-Ottawa Scale.

\begin{tabular}{|c|c|c|c|c|c|c|c|c|c|c|c|}
\hline \multirow[b]{2}{*}{ Authors and year } & \multicolumn{4}{|c|}{ Selection } & \multicolumn{2}{|c|}{ Comparability } & \multicolumn{3}{|c|}{ Exposure } & \multirow[b]{2}{*}{ Total score $=9$} & \multirow[b]{2}{*}{ (Refs.) } \\
\hline & 1 & 2 & 3 & 4 & 5 & 6 & 7 & 8 & 9 & & \\
\hline Cho et al 2007 & $\star$ & $\star$ & $\star$ & $\star$ & & & & $\star$ & $\star$ & 6 & (19) \\
\hline Zhang et al 2013 & $\star$ & $\star$ & $\star$ & & & $\star$ & & $\star$ & $\star$ & 6 & (16) \\
\hline Zhang et al 2012 & $\star$ & $\star$ & $\star$ & & & $\star$ & & $\star$ & $\star$ & 6 & (22) \\
\hline Yamaguchi et al 2001 & $\star$ & $\star$ & $\star$ & & & $\star$ & & $\star$ & $\star$ & 6 & $(20)$ \\
\hline Sirachainan et al 2012 & $\star$ & $\star$ & $\star$ & & & $\star$ & & $\star$ & $\star$ & 6 & (21) \\
\hline
\end{tabular}

For case-control studies, 1 indicates cases independently validated; 2 , cases are representative of population; 3 , hospital controls; 4 , controls have no history of cancer; 5 , study controls for age; 6 , study controls for additional factor (dihydropyrimidine dehydrogenase gene variants); 7 , ascertainment of exposure by blinded interview or record; 8 , same method of ascertainment used for cases and controls; 9 , non-respondents described.

\begin{tabular}{|c|c|c|c|c|c|}
\hline Study & $\begin{array}{l}\text { No. } \\
\text { case } \\
\text { SE }\end{array}$ & $\begin{array}{l}\text { of } 5-\mathrm{FU} \\
\text { es } \\
\text { Total }\end{array}$ & $\begin{array}{l}\text { No. o } \\
\text { cases } \\
\text { Non S }\end{array}$ & $\begin{array}{l}\text { on-5FU } \\
\text { Total }\end{array}$ & $\begin{array}{l}\log (\text { Ris\} } \\
\text { Ratio ) }\end{array}$ \\
\hline Cho 2007 & 21 & 67 & $\mathbf{0}$ & 100 & 4.1568 \\
\hline Sirachainan 2012 & 28 & 76 & $\mathbf{0}$ & 40 & 3.4128 \\
\hline Yamaguchi 2001 & 11 & 69 & $\mathbf{0}$ & 38 & 2.7371 \\
\hline Zhang 2012 & 162 & 167 & $\mathbf{0}$ & 193 & 0.9148 \\
\hline Zhang 2013 & 16 & 20 & 14 & 40 & 0.8267 \\
\hline Total $95 \%$ CI & & & & & \\
\hline
\end{tabular}

$\begin{array}{ll}\text { Weight } & \begin{array}{l}\text { Risk ratio } \\ \text { IV, Fixed } \\ \mathbf{9 5 \%} \text { CI }\end{array} \\ 0.4 \% & 63.87[3.94,1036.57] \\ 0.4 \% & 30.35[1.90,484.45] \\ 0.4 \% & 15.44[0.93,255.79] \\ 86.7 \% & 2.50[2.09,2.99] \\ 12.3 \% & 2.29[1.42,3.68] \\ 100 \% & 2.54[2.15,3.00]\end{array}$

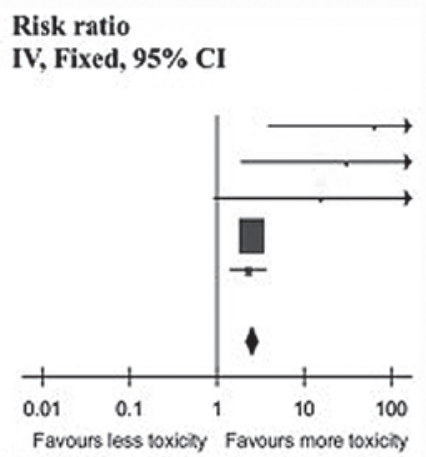

Figure 2. Risk of severe events (SE) (>grade 2) of 5-fluorouracil-based treatment associated with patients with the dihydropyrimidine dehydrogenase gene. $\mathrm{IV}$, inverse variance; df, degree of freedom; CI, confidence interval.

\section{Discussion}

The present results indicated that cancer patients with few DPYD polymorphisms identified from Asian populations are associated with an increased risk of severe toxicity of 5-FU-based chemotherapy. Of the 41 assessed polymorphisms, only 19 DPYD polymorphisms were formally associated with grade 3 or 4 toxicity in the meta-analysis. The associations were present in either 5-FU monotherapy or combination therapy. This finding appears to be inconsistent with a meta-analysis published in 2014 regarding genetic markers of toxicity in the QUASAR2 study, which reported that the associations were only present in 5-FU monotherapy (22). This may be due to differences in ethnicities as certain DPYD gene polymorphisms identified in Asian populations are different from those in the Western population; for example, $D P Y D^{*} 2 A$ (also known as $D P Y D$ : IVS14+1G $>\mathrm{A}$, c. $1905+1 \mathrm{G}>\mathrm{A}$ ) was not identified in the majority of the patients assessed from the Asian population. The inconsistent findings may be caused by a number of factors, such as geographic variability in the frequencies of rare gene polymorphisms, by sampling effects and possibly by variations in treatment regimens across studies or the individual patient response to toxicity. Individual patient response may be due to the complicated association between DPYD genotype and phenotype.
The data from the present meta-analysis showed that $D P Y D$ 1627A $>\mathrm{G}$ with a high $\mathrm{AF}$ was identified in cancer patients of China, Korea, Japan and Thailand (AF > 20\%) and $D P Y D 1896 \mathrm{~T}>\mathrm{C}$ was identified in Korean and Thai patients with AF $>14 \%$. The power to detect an association for these polymorphisms was $>75 \%$. Therefore, it could be characterized as a common polymorphism in these populations. For other polymorphisms, DPYD 85T >C and DPYD 2194G >A found in Chinese and Korean patients were low, resulting in $20 \%$ of suboptimal power to detect an association with severe toxicity.

According to the results of certain studies, a mortality rate of $0.5 \%$ and grades III-IV toxicity of $20-30 \%$ have been reported in patients treated with 5-FU for advanced cancer (17-21). Detection of the genetic polymorphism is thought to be a useful method for the prediction of severe toxicity and treatment outcome. Although the detection of the DPYD gene SNP cannot predict all the severe toxicity, $20 \%$ of all early 5 -FU-related toxicities could potentially be avoided (1). The identification of the remaining $80 \% 5-\mathrm{FU}$ toxicity must be reliant on the discovery and analysis of additional DPYD polymorphisms that can affect pharmacokinetics. The investigations of genetic markers of 5-FU-based regimens are being performed continuously. A recently published study identified that TYMS polymorphisms 5'VNTR2R/3R and 3'untranslated region 6 bp ins-del and DPYD 2846T $>\mathrm{A}$ and $* 2 \mathrm{~A}$ were significantly associated with grade 3 toxicity from the QUASAR2 
study (22). In addition, other genes, such as MTHFR and DPYD haplotypes, may also influence the responses to 5-FU $(1,13)$. There are no adequately published data from Asian populations that investigate in this respect. Therefore, no evidence was available for analysis in the present study.

Limitations in the systematic review included 2 points. Firstly, the number of clinical studies to explore the correlation between the DPYD variant and severe toxicity of 5-FU-based chemotherapy were limited, and the present result may be underestimated. Secondly, no studies reported the incidence or risk ratios of severe toxicity of 5-FU-associated with other genes, except $D P Y D$, in the Asian population.

In conclusion, the present meta-analysis suggested that several DPYD gene polymorphisms are associated with an increased risk of a severe toxic response to 5-FU in the Asian population. Therefore, the role of the DPYD gene polymorphisms used in predicting toxicity should be investigated continuously. Large and comprehensive studies are required in the near future prior to considering the use of DPYD as genetic markers of toxicity from 5-FU-based regimens in the Asian population.

\section{References}

1. Kristensen MH, Pedersen PL, Melsen GV, et al: Variants in the dihydropyrimidine dehydrogenase, methylenetetrahydrofolate reductase and thymidylate synthase genes predict early toxicity of 5-fluorouracil in colorectal cancer patients. J Int Med Res 38: $870-883,2010$

2. van Kuilenburg ABP, Meinsma R, Zonnenberg BA, Zoetekouw L, Baas F, Matsuda K, Tamaki N and van Gennip AH: Dihydropyrimidinase deficiency and severe 5-fluorouracil toxicity. Clin Cancer Res 9: 4363-4367, 2003.

3. Grem JL: 5-Fluorouracil: Forty-plus and still ticking. A review of its preclinical and clinical development. Invest New Drugs 18: 299-313, 2000

4. Twelves C, Wong A, Nowacki MP, Abt M, Burris H III, Carrato A, Cassidy J, Cervantes A, Fagerberg J, Georgoulias V, et al: Capecitabine as adjuvant treatment for stage III colon cancer. N Engl J Med 352: 2696-2704, 2005.

5. Boisdron-Celle M, Remaud G, Traore S, Poirier AL, Gamelin L, Morel A and Gamelin E: 5-Fluorouracil-related severe toxicity: A comparison of different methods for the pretherapeutic detection of dihydropyrimidine dehydrogenase deficiency. Cancer Lett 249: 271-282, 2007.

6. Saif MW, Choma A, Salamone SJ and Chu E: Pharmacokinetically guided dose adjustment of 5-fluorouracil: A rational approach to improving therapeutic outcomes. J Natl Cancer Inst 101: 1543-1552, 2009.

7. Van Kuilenburg AB, Vreken P, Beex LV, Meinsma R, Van Lenthe H, De Abreu RA and van Gennip AH: Heterozygosity for a point mutation in an invariant splice donor site of dihydropyrimidine dehydrogenase and severe 5-fluorouracil related toxicity. Eur J Cancer 33: 2258-2264, 1997.

8. Ezzeldin H and Diasio R: Dihydropyrimidine dehydrogenase deficiency, a pharmacogenetic syndrome associated with potentially life-threatening toxicity following 5-fluorouracil administration. Clin Colorectal Cancer 4: 181-189, 2004.
9. Schwab M, Zanger UM, Marx C, et al; German 5-FU Toxicity Study Group: Role of genetic and nongenetic factors for fluorouracil treatment-related severe toxicity: A prospective clinical trial by the German 5-FU Toxicity Study Group. J Clin Oncol 26: 2131-2138, 2008.

10. Afzal S, Gusella M, Vainer B, et al: Combinations of polymorphisms in genes involved in the 5-Fluorouracil metabolism pathway are associated with gastrointestinal toxicity in chemotherapy-treated colorectal cancer patients. Clin Cancer Res 17: 3822-3829, 2011.

11. Lecomte T, Ferraz JM, Zinzindohoué F, Loriot MA, Tregouet DA, Landi B, Berger A, Cugnenc PH, Jian R, Beaune $\mathrm{P}$, et al: Thymidylate synthase gene polymorphism predicts toxicity in colorectal cancer patients receiving 5-fluorouracil-based chemotherapy. Clin Cancer Res 10: 5880-5888, 2004.

12. Chazal M, Etienne MC, Renée N, Bourgeon A, Richelme H and Milano G: Link between dihydropyrimidine dehydrogenase activity in peripheral blood mononuclear cells and liver. Clin Cancer Res 2: 507-510, 1996.

13. Diasio RB: Dihydropyrimidine dehydrogenase (DPD) deficiency: A 5-fluorouracil-associated pharmacogenomic syndrome. Horiz Cancer Ther 4: 9-21, 2003.

14. Johnson MR, Wang K and Diasio RB: Profound dihydropyrimidine dehydrogenase deficiency resulting from a novel compound heterozygote genotype. Clin Cancer Res 8: 768-774, 2002.

15. Dolegowska B, Ostapowicz A, Stanczyk-Dunaj M and Blogowski W: Spectrophotometric methods as a novel screening approach for analysis of dihydropyrimidine dehydrogenase activity before treatment with 5 -fluorouracil chemotherapy. J Physiol Pharmacol 63: 411-421, 2012.

16. Zhang X, Sun B and Lu Z: Evaluation of clinical value of single nucleotide polymorphisms of dihydropyrimidine dehydrogenase gene to predict 5 -fluorouracil toxicity in 60 colorectal cancer patients in China. Int J Med Sci 10: 894-902, 2013.

17. Wells GA, Shea B, O'Connell D, et al: The Newcastle-Ottawa Scale (NOS) for assessing the quality of nonrandomised studies in meta-analyses. 3rd Symposium on Systematic Reviews: Beyond the Basics, Oxford, UK, 2000. http://www. ohri.ca/programs/clinical_epidemiology/oxford.htm. Accessed August 12, 2014.

18. Cho HJ, Park YS, Kang WK, Kim JW and Lee SY: Thymidylate synthase (TYMS) and dihydropyrimidine dehydrogenase (DPYD) polymorphisms in the Korean population for prediction of 5-fluorouracil-associated toxicity. Ther Drug Monit 29: 190-196, 2007.

19. Yamaguchi K, Arai Y, Kanda Y and Akagi K: Germline mutation of dihydropyrimidine dehydrogenese gene among a Japanese population in relation to toxicity to 5-Fluorouracil. Jpn J Cancer Res 92: 337-342, 2001.

20. Sirachainan E, Reungwetwattana T, Wisetpanit Y, et al: Pharmacogenetic study of 5-FU-related severe toxicity in Thai cancer patients: A novel SNP detection. J Pharmacogenomics Pharmacoproteomics 3: 1-4, 2012.

21. Zhang XP, Bai ZB, Chen BA, Feng JF, Yan F, Jiang Z, Zhong YJ, Wu JZ, Chen L, Lu ZH, et al: Polymorphisms of dihydropyrimidine dehydrogenase gene and clinical outcomes of gastric cancer patients treated with fluorouracil-based adjuvant chemotherapy in Chinese population. Chin Med J (Engl) 125: 741-746, 2012.

22. Rosmarin D, Palles C, Church D, Domingo E, Jones A, Johnstone E, Wang H, Love S, Julier P, Scudder C, et al: Genetic markers of toxicity from capecitabine and other fluorouracilbased regimens: Investigation in the QUASAR2 study, systematic review, and meta-analysis. J Clin Oncol 32:1031-1039, 2014. 